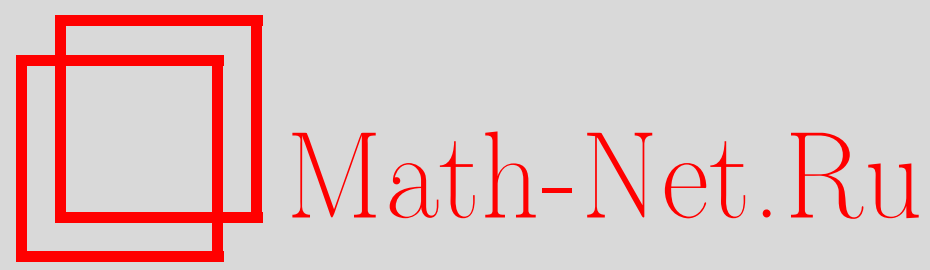

К. Ю. Горбунов, Одно обобщение теоремы Гильберта о базисе, Матем. заметки, 2003, том 74, выпуск 4, 508-516

DOI: https://doi.org/10.4213/mzm286

Использование Общероссийского математического портала Math-Net.Ru подразумевает, что вы прочитали и согласны с пользовательским соглашением http://www. mathnet.ru/rus/agreement

Параметры загрузки:

IP: 3.82 .47 .9

26 апреля 2023 г., 12:50:49

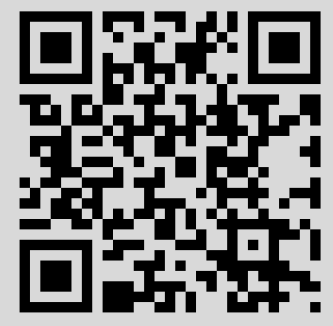




\title{
ОДНО ОБОБЩЕНИЕ ТЕОРЕМЫ ГИЛЬБЕРТА О БАЗИСЕ
}

\author{
К. Ю. Горбунов
}

\begin{abstract}
Предлагается обобщение геометрической формы теоремы Гильберта о базисе, утверждающее, что для каждого хорошо описываемого (в некотором смысле) семейства многочленов существует такое число $C$, что для всюду плотного (в некотором смысле) подсемейства $P$ этого семейства и любой точки $a$ если первые $C$ многочленов любой последовательности из $P$ равны нулю в точке $a$, то и все ее многочлены равны нулю в $a$.

Библиография: 4 названия.
\end{abstract}

Геометрическая форма теоремы Гильберта о базисе утверждает, что последовательность вложенных алгебраических многообразий ${ }^{1}$ стабилизируется. Иначе говоря, для любой последовательности $S$ многочленов от переменных $x_{1}, \ldots, x_{k}$ существует такое число $C$, что для любой точки пространства $a=\left\langle x_{1}^{*}, \ldots, x_{k}^{*}\right\rangle$ если первые $C$ многочленов в последовательности $S$ равны нулю в точке $a$, то и все многочлены из $S$ в этой точке равны нулю (в этом случае будем говорить, что число $C$ обслуживает последовательность $S$ ). Эта форма является следствием обычной теоремы Гильберта о базисе, а если ограничиться радикальными идеалами (т.е. идеалами, замкнутыми относительно извлечения корней), то эквивалентна ей. Мы будем обобщать эту теорему в направлении, где утверждается существование одного $C$, обслуживающего целое семейство последовательностей многочленов. Конечно, чтобы обобщение было нетривиальным, семейство должно, во-первых, содержать многочлены сколь угодно большой степени, а во-вторых, не быть семейством многочленов от конечного числа выражений. Учитьвая это, дадим следующее определение.

ОПРЕДЕЛЕНИЕ. Квазимногочленом от переменных $x_{1}, x_{2}, \ldots, x_{k}$ будем называть синтаксическое выражение - многочлен от $x_{1}, x_{2}, \ldots, x_{k}$ и от выражений $F\left(x_{1}\right), F\left(x_{2}\right), \ldots, F\left(x_{k}\right)$, а также от производных - выражений $F^{\prime}\left(x_{1}\right), \ldots, F^{\prime}\left(x_{k}\right), \ldots$, $F^{(i)}\left(x_{1}\right), \ldots, F^{(i)}\left(x_{k}\right), \ldots,{ }^{2}$ например, $x_{2} F\left(x_{1}\right) F^{\prime \prime \prime}\left(x_{1}\right)+x_{3} F^{\prime \prime}\left(x_{2}\right) F^{\prime \prime}\left(x_{2}\right)$.

Пусть дана последовательность $S=q_{1}, q_{2}, \ldots$ квазимногочленов. Если $p=p_{n} x^{n}+$ $p_{n-1} x^{n-1}+\cdots+p_{1} x+p_{0}-$ многочлен и мы подставим в $S$ всюду вместо $F\left(x_{i}\right), i=1, \ldots, k$,

Работа выполнена при частичной поддержке гранта Российского фонда фундаментальных исследований, грант № 01-01-01028.

${ }^{1}$ Здесь и далее мы будем понимать под алгебраическим многообразием совместное множество нулей конечной системы многочленов в афффинном пространстве над полем действительных или комплексных чисел.

${ }^{2}$ Обычно квазимногочленом называют многочлен от переменных и их экспонент. Мы используем естественное обобщение этого понятия. 
многочлен $p\left(x_{i}\right)$ (и соответствующие многочлены вместо производных), то получим последовательность многочленов $S(p)=q_{1}(p), q_{2}(p), \ldots$ Конечно, мы не можем гарантировать существование одного числа $c$, которое обслуживает последовательность $S(p)$ при любом многочлене $p$. Действительно, если $S=F(x), F^{\prime}(x), F^{\prime \prime}(x), \ldots$, то для $p=x^{n}$ в точке $x=0$ первые $n$ членов $S$ равны нулю, а $(n+1)$-й не равен. Однако следующая теорема утверждает, что существует одно число $c$, которое обслуживает $S(p)$ для всюду плотного (в некотором смысле) множества многочленов $p$. Точнее, скажем, что для почти всех многочленов выполнено некоторое свойство $P$, если для любого достаточно большого $n$ и любых $p_{0}, \ldots, p_{n}, \varepsilon$ существуют $\varepsilon_{1}$ и $\bar{p}_{0}, \ldots, \bar{p}_{n}$, где $\left|\bar{p}_{i}-p_{i}\right|<\varepsilon$, такие, что для любых $\bar{p}_{0}^{*}, \ldots, \bar{p}_{n}^{*}$, где $\left|\bar{p}_{i}^{*}-\bar{p}_{i}\right|<\varepsilon_{1}$, многочлен $\bar{p}_{n}^{*} x^{n}+\cdots+\bar{p}_{0}^{*}$ удовлетворяет свойству $P$.

ТЕОремА. Для любой бесконечной последовательности $S$ квазимногочленов от $x_{1}, x_{2}, \ldots, x_{k}$ существует такое число $c$, что для почти всех многочленов $p$ для любой точки $a=\left\langle x_{1}^{*}, \ldots, x_{k}^{*}\right\rangle$ либо все многочлены в последовательности $S(p)$ в $a$ равны нулю, либо некоторый многочлен из $S(p)$ с номером не больше с не равен нулю в точке $а$.

ДокАЗАТЕЛЬСТво. Сначала докажем вспомогательную лемму, дающую для любого квазимногочлена $q$ необходимое и достаточное условие того, что если многочлен $q(p)$ равен нулю в точке $a$, то можно сколь угодно мало сдвинуть (не зависящее от степени $p$ ) число коэффициентов многочлена $p$ при младших степенях, чтобы для получившегося многочлена $\bar{p}$ многочлен $q(\bar{p})$ не равнялся 0 в точке $a$.

Лемма 1. Пусть $q$ - квазимногочлен от $x_{1}, \ldots, x_{k}$. Тогда существует такое число $m$, что для любого многочлена $p=p_{n} x^{n}+\cdots+p_{0}$ степени $n>m$ и любой точки $a=\left\langle x_{1}^{*}, \ldots, x_{k}^{*}\right\rangle$ следующие два утвержсдения әквивалентны:

1) если многочлен $q(p)$ равен нулю в точке $a$, то для любого $\varepsilon>0$ существуют такие числа $\bar{p}_{0}, \ldots, \bar{p}_{m}$, что $\left|\bar{p}_{i}-p_{i}\right|<\varepsilon, i=0, \ldots, m$, и многочлен $q(\bar{p})$, где $\bar{p}=p_{n} x^{n}+\cdots+p_{m+1} x^{m+1}+\bar{p}_{m} x^{m}+\cdots+\bar{p}_{0}$, не равен нулю в точке $a$;

2) если подставить в $q$ значения $x_{1}^{*}, \ldots, x_{k}^{*}$, то получится вырахсение - ненулевой полином от $F\left(x_{i}^{*}\right), F^{\prime}\left(x_{i}^{*}\right), F^{\prime \prime}\left(x_{i}^{*}\right), \ldots$.

ДокАЗАтЕльство. Импликация 1) $\Rightarrow 2$ ) очевидна. Докажем импликацию 2) $\Rightarrow 1$ ). Назовем типом точки а информацию о том, какие из ее координат $x_{i}^{*}$ попарно равны. Очевидно, достаточно доказать лемму для точек одного произвольного типа. Отождествив в $q$ выражения $F\left(x_{i}\right)$ и $F\left(x_{j}\right)$, если $x_{i}^{*}=x_{j}^{*}$, будем считать, что все $x_{i}^{*}$ различны.

Возьмем $m \geqslant \sum_{i=1}^{k}\left(\alpha_{i}+1\right)$, где $\alpha_{i}$ - максимальный порядок производной выражения $F\left(x_{i}\right)$, входящей в $q$. Зафиксируем значения $F^{(j)}\left(x_{i}^{*}\right)=\beta_{i j}, j=0, \ldots, \alpha_{i}$, так, чтобы значение $q$ было ненулевым в точке $a$ (по условию 2 ) это возможно). Из теории интерполящии с кратными узлами (см., например, $[1$, глава $3, \S 6])$ известно, что существует многочлен $g$ степени не более $m$ такой, что для всех $i$ и $j$ выполнено равенство $g^{(j)}\left(x_{i}^{*}\right)=\beta_{i j}$. Отсюда следует, что если в $q$ подставить вместо $F$ многочлен степени $m$ с неопределенными коэффициентами, то получится ненулевой (в точке $a$ ) многочлен $q_{m}$ от этих коэффициентов. Если же подставить вместо $F$ многочлен любой степени $n>m \mathrm{c}$ неопределенными коэффициентами, то полученный многочлен $q_{n}$ будет иметь вид $q_{m}+\bar{q}$, причем в каждом члене $\bar{q}$ присутствует хотя бы один неопределенньй коэффициент $p_{i}$, где $i>m$. Таким образом, и в этом случае многочлен $q_{n}$ ненулевой. Теперь утверждение леммы следует из того геометрически очевидного факта, что ненулевой полином 
задает в пространстве алгебраическое многообразие меньшей, чем само пространство, размерности, и всегда можно сдвинуться из его корня на сколь угодно малое расстояние, чтобы сойти с этого многообразия (строгое рассуждение легко провести индукцией по числу переменных). Лемма 1 доказана.

Продемонстрируем на примере основную идею доказательства теоремы.

ПРимеР. Пусть последовательность $S$ начинается так: $y x^{2}, x^{3}+z, \ldots$, и мы хотим упростить ее второй член, используя информацию о том, что первьй член равен нулю. Тогда естественно рассмотреть два случая:

1) $y=0-$ в этом случае второй член упростить не удается, зато первый член можно заменить на $y$;

2) $y \neq 0$ - в этом случае второй член можно заменить на $z$.

Таким образом, вместо одной последовательности возникает две:

$$
y=0, x^{3}+z=0, \ldots \quad \text { и } \quad\left(y x^{2}=0 \& y \neq 0\right), z=0, \ldots
$$

Эти две последовательности естественно представлять в виде дерева с добавленным (пустьм) корнем и двумя ветвями. Тогда перебор возможных случаев (как было выше) соответствует расщеплению одной вершины на несколько.

Перейдем к формальному изложению доказательства.

Напомним, что типом точки назьвается информация о том, какие из ее координат попарно равны. Пусть $t_{1}, \ldots, t_{d}$ - все возможные типы. Для каждого типа $t$ независимо проведем обработку последовательности $S$, состоящую в следующем. Отождествим равные переменные и соответствующие им выражения $F\left(x_{i}\right), F^{\prime}\left(x_{i}\right), \ldots$, заменив все переменные из класса равных переменных на переменную из этого класса с наименьшим индексом. Получим последовательность $S_{t}$.

Будем строить бесконечное (но с конечными степенями вершин) дерево $T$ (представляем его "растущим" вверх), каждая вершина которого помечена конечным числом равенств типа $q=0$ и неравенств типа $q \neq 0$, где $q$ - ненулевой квазимногочлен (разметка вершины может быть и пустой). Вначале в качестве $T$ возьмем дерево $T_{t}$, в котором любая вершина имеет ровно одного "сьна" и для каждого $i$-я снизу вершина помечена приравненньп к нулю $i$-м членом последовательности $S_{t}$. Путь (конечный или бесконечный), начинающийся в корне и идущий все время вверх, будем назьвать корневы. Компонентами разметки будем называть переменные $x_{1}, \ldots, x_{k}$ и выражения $F\left(x_{1}\right), \ldots, F\left(x_{k}\right), F^{\prime}\left(x_{1}\right), \ldots, F^{\prime}\left(x_{k}\right), \ldots$

В конце конструкции дерево $T$ будет удовлетворять следующим четырем основным свойствам.

1. Если для некоторого конечного корневого пути $\gamma_{1}$ в $T_{t}$ и некоторой точки $a$ выполняются все равенства на пути $\gamma_{1}$, то в $T$ существует корневой путь $\gamma_{2}$ той же длины, на котором удовлетворяется вся разметка (т.е. вьполняются и равенства и неравенства) в точке $a$.

2. Если для некоторого конечного корневого пути $\gamma$ в $T$ и некоторой точки $a$ удовлетворяется вся разметка на пути $\gamma$, то в точке $a$ выполняются все равенства на корневом пути той же длины в $T_{t}$.

3. Разметка любой вершины в $T$ либо пуста, либо содержит хотя бы одно равенство.

4. Для любой компоненты на любом бесконечном корневом пути в $T$ есть лишш конечное число вершин, в разметке которых встречается эта компонента. 
Упорядочим компоненты разметки так:

$$
x_{1}, \ldots, x_{k}, \quad F\left(x_{1}\right), \ldots, F\left(x_{k}\right), \quad F^{\prime}\left(x_{1}\right), \ldots, F^{\prime}\left(x_{k}\right), \ldots
$$

Каж дую компоненту обрабатываем описанньм далее образом. Перед началом обработки очередной компоненты кроме первых трех основных свойств выполнено следующее “промежуточное" свойство.

4*. На любом бесконечном корневом пути в дереве имеется лишь конечное число вершин, разметка которых содержит уже обработанные компоненты или неравенства.

Будем назьвать активными те вершины, в текущей разметке которых не встречается уже обработанньх компонент, но встречается обрабатьваемая компонента. Опишем ее обработку. Пусть, для примера, обрабатьваемая компонента $-F\left(x_{1}\right)$. Будем рассматривать все левые части равенств как полиномы от одной переменной - компоненты $F\left(x_{1}\right)$ с коэффициентами - полиномами от остальных компонент (под степенью полинома понимаем максимальную входящую в него степень $\left.F\left(x_{1}\right)\right)$. Обрабатьваем лишь активные вершины дерева, причем начинаем с нижних, так что перед обработкой очередной вершины $v$ все активные вершины ниже нее уже обработаны, и в каждой из них все коэффициенты при степенях $F\left(x_{1}\right)$ в равенствах разметки являются левьми частями некоторых неравенств разметки.

Обрабатываем $v$ следующим образом. Если степени всех полиномов, стоящих в левых частях равенств в разметке $v$, меньше, чем степени всех полиномов в разметке активных вершин на пути от $v$ до корня, то расшепляем вершину $v$ на конечное число вершин с тем же родителем. Каждая из них соответствует одному указанию на то, какие коэффициенты в многочленах из левых частей равенств разметки $v$ равны нулю, а какие не равны (перебираются все случаи). В случае, когда коэффициент равен нулю, это равенство добавляется в разметку образуемой вершины, а из полинома вычеркивается соответствующий член, когда не равен - добавляем в разметку это неравенство. Так, если разметкой $v$ является

$$
F\left(x_{2}\right) F^{2}\left(x_{1}\right)+F\left(x_{3}\right) F\left(x_{1}\right)+1=0
$$

то вместо $v$ будет 8 вершин с разметками

1) $F\left(x_{2}\right) \neq 0, F\left(x_{3}\right) \neq 0,1 \neq 0, F\left(x_{2}\right) F^{2}\left(x_{1}\right)+F\left(x_{3}\right) F\left(x_{1}\right)+1=0$;

2) $F\left(x_{2}\right)=0, F\left(x_{3}\right) \neq 0,1 \neq 0, F\left(x_{3}\right) F\left(x_{1}\right)+1=0$;

3) $F\left(x_{2}\right) \neq 0, F\left(x_{3}\right)=0,1 \neq 0, F\left(x_{2}\right) F^{2}\left(x_{1}\right)+1=0$;

4) $F\left(x_{2}\right) \neq 0, F\left(x_{3}\right) \neq 0,1=0, F\left(x_{2}\right) F^{2}\left(x_{1}\right)+F\left(x_{3}\right) F\left(x_{1}\right)=0$;

5) $F\left(x_{2}\right) \neq 0, F\left(x_{3}\right)=0,1=0, F\left(x_{2}\right) F^{2}\left(x_{1}\right)=0$;

6) $F\left(x_{2}\right)=0, F\left(x_{3}\right) \neq 0,1=0, F\left(x_{3}\right) F\left(x_{1}\right)=0$;

7) $F\left(x_{2}\right)=0, F\left(x_{3}\right)=0,1 \neq 0,1=0$;

8) $F\left(x_{2}\right)=0, F\left(x_{3}\right)=0,1=0$.

Выше каждой из этих вершин располагаем копию множества вершин, находившегося выше $v$. Описанная операция расшепления вершины сохраняет вьполненность основного свойства 1 (поскольку рассматриваются все случаи), основного свойства 2 (поскольку из истинности разметки новой вершины следует истинность разметки старой) и основного свойства 3 (поскольку неравенство нулю некоторого коэффициента полинома оставляет этот полином ненулевым). 
Теперь рассмотрим случай, когда в некоторой вершине $u$ ниже $v$ стоит равенство с полиномом $p_{u}$, а в вершине $v$ - с полиномом $p_{v}$, причем $n$ - степень $v$ - не меньше $m$ - (ненулевой) степени $p_{u}$ (будем считать, что вершина $u$ выбрана так, что $m$ минимально). Для каждого такого $p_{v}$ совершаем следующие действия. Домножим $p_{v}$ на $(n-m+1)$-ю степень старшего коэффициента $p_{u}$, чтобы при делении “уголком" полученного полинома на $p_{u}$ не возникали дроби. Выполнив это деление, получим равенство $p_{m}^{n-m+1} p_{v}=q p_{u}+r$, где $p_{m}-$ старший коэффициент $p_{u}$, многочлен $r-$ так назьваемьй nсевдоостаток (или модифицированньй остаток), степень $r$ строго меньше степени $p_{u}$ (отметим, что понятие псевдоостатка использовалось китайским математиком Ву Вень Цунем для алгоритмического доказательства теорем евклидовой геометрии (см., например, $[2$, гл. $6, \S 5])$, а также Мучником для нового более простого доказательства теоремы Тарского об элиминации кванторов, см. [3, гл. 3, п. 8]). Например, если

$$
p_{v}=F^{2}\left(x_{3}\right) F^{3}\left(x_{1}\right)-F\left(x_{1}\right), \quad p_{u}=F^{3}\left(x_{3}\right) F\left(x_{1}\right)-2,
$$

TO

$$
\begin{aligned}
\left(F^{3}\left(x_{3}\right)\right)^{3} p_{v}= & \left(F^{8}\left(x_{3}\right) F^{2}\left(x_{1}\right)+2 F^{5}\left(x_{3}\right) F\left(x_{1}\right)+4 F^{2}\left(x_{3}\right)-F^{6}\left(x_{3}\right)\right) p_{u} \\
& +\left(8 F^{2}\left(x_{3}\right)-2 F^{6}\left(x_{3}\right)\right) .
\end{aligned}
$$

Поскольку в разметке вершины $u$ стоит неравенство $p_{m} \neq 0$, имеем $p_{v}=0$ тогда и только тогда, когда $r=0$. Если $r$-ненулевой многочлен, то в разметке вершины $v$ заменяем $p_{v}$ на $r$, а если нулевой - убираем из этой разметки равенство $p_{v}=0$. При этом сохраняется вьполненность основных свойств 1 и 2 (очевидно) и основного свойства 3 (поскольку вершина $v$ активна, а значит в ее разметке нет неравенств). Выполнив все возможные деления и замены делимых на остатки, мы свели ситуацию к уже рассмотренному случаю, после чего проводится описанное ранее расщепление вершины $v$.

Легко видеть, что после обработки компоненты (т.е. описанной обработки счетного множества вершин) остаются вьполненными основные свойства 1, 2 и 3 . Выполнено и "промежуточное" свойство 4 *, поскольку вдоль корневого пути степени полиномов в равенствах разметки строго убывают, а любое неравенство стоит в разметке вершины лишь вместе с равенством, содержащим ту же компоненту.

При обработке счетного числа компонент вершины фиксированной высоты активны лишь конечное число раз. Поэтому можно говорить о предельном дереве $T$, в котором, очевидно, вьполнены все 4 основных свойства. Из свойства 4 вытекает следующая лемма. Назовем рангом вершины $v$ (обозначение: $\operatorname{rang}(v)$ ) минимальный порядок производных, встречаюшихся в разметке вершины $v$, а если в разметке присутствует хотя бы одна переменная $x_{i}$ (некак аргумент $F$ ) или разметка пуста, то считаем ранг равным -1 . Ранг множества вершин $M$ определим как $\operatorname{rang}(M)=\min _{v \in M} \operatorname{rang}(v)$ (считаем, что $\operatorname{rang}(\varnothing)=\infty)$. Вершину, из которой есть бесконечньй путь вверх по вершинам с пустой разметкой, назовем крайней (сама она может иметь непустую разметку).

ЛЕмма 2. Для любых чисел $r, h$ в предельном дереве $T$ существует конечное подмножество вершин $M$ со следующими свойствами:

1) $\operatorname{rang}(M)>r$, выссота всех вершин из $M$ больше $h$;

2) существует такое число $h_{1}$, что любой бесконечный корневой путь в $T$ либо пересекает $M$ ровно в одной вериине, либо проходит через крайнюю вершину, лежсашую на высоте не больше $h_{1}$. 
ДокАЗАтЕльство. Заметим, что сушествует такая высота $h_{1}>h$, что любой бесконечньй корневой путь на отрезке между высотами $h$ и $h_{1}$ либо проходит через крайнюю вершину, либо через вершину ранга больше $r$. Действительно, иначе по свойству компактности (лемма Кёнига) существовал бы бесконечньй корневой путь, который проходил бы через бесконечное число вершин ограниченного ранга с непустой разметкой, что противоречит основному свойству 4 . Таким образом, множество $M$ составляется так: для каждого корневого пути заносим в $M$ первую из его вершин ранга больше $r$ на высотах между $h$ и $h_{1}$ (если она есть). Лемма 2 доказана.

Напомним, что для каждого типа точки мыполучили свое обработанное дерево. Упорядочим эти деревья произвольным образом: $T_{1}, T_{2}, \ldots, T_{d}$. В каждом из $T_{i}$ построим $k+1$ конечных множеств вершин (будем назьвать эти множества ярусами) $M_{i}^{1}, M_{i}^{2}$, $\ldots, M_{i}^{k+1}$ со следующими свойствами (введем линейньй порядок: скажем, что $M_{i}^{j} н u-$ же $M_{s}^{l}$, если либо $i<s$, либо $i=s$ и $j<l$ ).

1. Для любого яруса $M$, во-первых, $\operatorname{rang}(M)>0$, а во-вторых, если ниже есть непустые ярусы, то $\operatorname{rang}(M)>\operatorname{rang}(\bar{M})+m$, где $\bar{M}-$ ближайший к $M$ снизу непустой ярус, а $m$ - максимальное из тех чисел $m$, которые по лемме 1 соответствуют многочленам из равенств разметки яруса $\bar{M}$ (присутствие там равенств следует из основного свойства 4).

2. Высоты всех вершин каждого яруса больше всех высот вершин предыдуших ярусов.

3. Существует такое число $h$, что в каждом $T_{i}$ любой бесконечный корневой путь либо пересекает все ярусы $M_{i}^{1}, \ldots, M_{i}^{k+1}$, причем каждый ровно в одной вершшне, либо проходит через крайнюю вершину, лежащую на высоте не больше $h$.

Очевидно, что, используя лемму 2 , можно построить требуемые $M_{i}^{j}$ по одному, начиная с самых нижних. Свойство 1 ярусов выражает основную идею наших дальнейших действий: мы собираемся осуществлять сдвиг коэффициентов $p_{0}, p_{1}, \ldots, p_{m}$ в $F$, который бы менял функцию, стоящую в левой части равенства разметки вершины из некоторого яруса, но оставлял бы неизменными все функции из разметки более высоких ярусов.

Зафиксируем степень $n$ больше рангов всех ярусов. Возьмем произвольный многочлен $p$ степени $n$. В разметках ярусных вершин подставим вместо $F$ полином $p$ (и соответствующие выражения вместо производных). Зафиксируем по одному равенству в разметке каждой ярусной вершины $v$. Это равенство определяет алгебраическое многообразие (будем обозначать его как $R(v)$ ) в пространстве переменных $x_{1}, \ldots, x_{k(i)}$ $\left(k(i) \leqslant k\right.$ из-за отождествления равных переменных, здесь $\left.v \in T_{i}\right)$. Если $u \in M_{i}^{j}$, $v \in M_{i}^{l}, j<l$, и $v$ является потомком вершины $u \neq v$ в дереве $T_{i}$, то скажем, что многообразие $R(v)$ - потомок многообразия $R(u)$.

Будем перебирать ярусы, начиная с самых высоких, и для каждой их вершины $v$ осуществлять сдвиги коэффициентов (текущего) многочлена $p$ так, чтобы многообразие $R(v)$ находилось в как можно более общем положении относительно совокупности своих потомков. Точнее, для каждого пути из $v$ вверх рассматриваем алгебраическое многообразие $R$, равное пересечению всех потомков многообразия $R(v)$ на этом пути. Из алгебраической геометрии известно (см., например, [4, гл. $1, \S 3$, теорема 1] или [2, гл. $4, \S 6$, теорема 2$]$ ), что любое многообразие является объединением конечного числа неприводимых (т.е. не представимых в виде объединения двух непустых многообразий) 
многообразий, называемых его неприводимыми компонентами. Будем рассматривать неприводимые над полем комплексных чисел компоненты многообразия $R$, причем лишь такие, которые содержат хотя бы одну точку с попарно различными координатами (напомним, что нас интересуют только точки, у которых все координаты $x_{i}$ попарно различны, иначе за эти точки “отвечает" другое дерево). Мы хотим сдвинуть коэффициенты $p$ в малой окрестности так, чтобы $R$ осталось неподвижным, а каждая его неприводимая компонента $R^{*}$ указанного вида пересекалась с многообразием $R(v)$ по многообразию меньшей, чем у $R$, размерности. Геометрически ясно, что для этого достаточно сдвинуть $R(v)$ с произвольной точки компоненты $R^{*}$, причем сдвинуть столь мало, чтобы размерности пересечений других многообразий (из конечного числа) остались меньшими размерностей самих многообразий.

По лемме 1 при достаточно большом $n$ возможен сдвиг из любой точки с попарно различными координатами. Действительно, из их различности и положительности ранга вершины $v$ вытекает выполненность условия 2) леммы 1. Из свойства 1 ярусов следует неподвижность многообразия $R$ и всех многообразий из более высоких ярусов. Эта неподвижность гарантирует, что сдвиг каждьй раз можно делать столь мальм, чтобы все ранее достигнутые “общие положения" многообразий таковьми и остались. Из алгебраической геометрии известно (см., например, [4, гл. $1, \S 6$, теорема 1] или [2, гл. 9, $\S 4$, предложение 10]), что если произвольное многообразие $X$ является строгим подмножеством неприводимого многообразия $Y$, то размерность $X$ строго меньше размерности $Y$. Поэтому каждый раз, когда мы пересекаем (сдвинутое) текущее многообразие с пересечением его потомков на пути, размерность пересечения уменьшается. Осуществив описанные сдвиги коэффициентов, получим из $p$ многочлен $\bar{p}$.

Возьмем требуемое в формулировке теоремы число $c$ больше числа $h$ из свойства 3 ярусов. Докажем, что это с обслуживает $S(\bar{p})$. Действительно, пусть первые $c$ равенств последовательности $S$ вьполняются в точке $a$ типа $t$. Тогда первые $c$ равенств последовательности $S_{t}$ вьполняются в точке $a_{t}$, полученной из $a$ отождествлением равных координат. Пусть типу $t$ соответствует дерево $T_{i}$. По основному свойству 1 в $T_{i}$ есть корневой путь $\gamma$ длины $c$ с удовлетворяющейся в точке $a_{t}$ разметкой. Для него есть две возможности.

Случай 1. Пусть $\gamma$ пересекает все ярусы $M_{i}^{1}, \ldots, M_{i}^{k+1}$, каждьй в одной вершине. Обозначим эти вершины, соответственно, $v_{1}, \ldots, v_{k+1}$. По построению, последовательность размерностей тех неприводимых компонент многообразий

$$
R\left(v_{k+1}\right), \quad R\left(v_{k+1)} \cap R\left(v_{k}\right), \ldots, \quad R\left(v_{k+1}\right) \cap R\left(v_{k}\right) \cap \cdots \cap R\left(v_{1}\right),\right.
$$

в которьх находится точка $a_{t}$ (с попарно неравными координатами), строго убывает. Поскольку размерность пространства не превьшает $k$, этот случай невозможен.

Случай 2. Пусть $\gamma$ проходит через крайнюю вершину $v$. Рассмотрим бесконечньй корневой путь $\bar{\gamma}$, совпадающий с $\gamma$ до $v$, а дальше идущий по вершинам с пустой разметкой. По основному свойству 2 , примененному к произвольному началу пути $\bar{\gamma}$, все равенства последовательности $S_{t}$ выполняются в точке $a_{t}$, а значит все равенства последовательности $S$ вьполняются в точке $a$.

Для завершения доказательства теоремы нам осталось показать, что можно добиться, чтобы число $c$ обслуживало не только сам $\bar{p}$, но и некоторую его окрестность. Покажем, как осуществить сколь угодно мальй сдвиг произвольных коэффищиентов многочлена $p$, чтобы полученньй $\bar{p}$ удовлетворял этому свойству. Для выбранной ранее 
степени $n$ подставим в разметку всех деревьев $T_{i}$ вместо $F$ многочлен степени $n$ с неопределенньми коэффициентами. Для каждого дерева и каждого корневого пути, пересекающего все ярусы в этом дереве в вершинах $v_{1}, \ldots, v_{k}$, напишем формулу $\Phi_{i}^{\gamma}$, утверждающую, что пересечение всех многообразий $R\left(v_{i}\right)$ пусто. Эта формула имеет свободными переменными неопределенные коэффициенты многочлена $p$. По теореме Тарского об элиминации кванторов (верной как над полем действительных чисел, так и над полем комплексных чисел, см., например, [3, гл. 3, п. 8]) существует эквивалентная ей бескванторная формула. Сдвиг коэффициентов $p$ осуществим так, чтобы все полиномы от коэффициентов во всех полученных бескванторных формулах имели ненулевые значения. Покажем, что при этом все формулы $\Phi_{i}^{\gamma}$ станут истинными. Действительно, если бы некоторая формула была ложна, то можно было бы осуществить описанные ранее сколь угодно малые сдвиги коэффициентов (для приведения соответствующих многообразий в общее положение) и формула стала бы истинной. Это противоречит тому, что при малых сдвигах не меняются знаки в полиномах эквивалентной бескванторной формулы. По этой же причине все формулы $\Phi_{i}^{\gamma}$ истинны не только для самого многочлена $\bar{p}$, но и для всех многочленов в его малой коэффициентной окрестности, что и требовалось. Теорема доказана.

ЗАмЕчАниЕ 1. Легко видеть, что в формулировке теоремы вместо последовательности $S$ можно сразу рассматривать (размеченное равенствами) произвольное дерево $S$. Тогда утверждение теоремы будет состоять в том, что существует такое $c$, что для почти всех $p$ для любой точки либо существует бесконечный корневой путь, на котором выполняются все равенства, либо на любом бесконечном корневом пути некоторое равенство на высоте не более $c$ не вьполняется. В доказательстве основное свойство 2 следует переформулировать так: если для некоторого конечного корневого пути $\gamma$ в $T$ и некоторой точки $a$ удовлетворяет вся разметка на пути $\gamma$, то существует корневой путь той же длины в $T_{t}$, на котором выполняются все равенства в точке $a$ (а именно, прообраз пути $\gamma$ относительно расщепления вершин). В остальном доказательство аналогично.

ЗАмЕчАниЕ 2. Доказанную теорему нетрудно обобщить на случай, когда функция $F$ имеет не один аргумент, а фиксированное конечное число аргументов, а в квазимногочленах допускаются частные производные (например $\partial^{4} F /\left(\partial x_{1} \partial x_{2} \partial x_{2} \partial x_{3}\right)$; вместо $F$ теперь подставляются многочлены от соответствующего числа переменных). Для удобства можно считать, что производные по переменным всегда берутся в порядке возрастания номеров этих переменных. Единственное существенное усложнение доказательства состоит в том, что в доказательстве леммы 1 вместо упомянутого результата из теории кратной интерполяции следует использовать

УТВЕРЖДЕНИЕ. Любой конечный набор условий, т.е. значений функиии и ее частных производных в заданных точках, удовлетворяется на полиноме, степень которого зависит только от числа переменных, числа точек и максимального порядка встречающихся в условиях производных.

Доказать его можно, например, следующим образом.

Назовем условие равенства заданному значению частной производной суммарного порядка $i$ (соответственно, порядка $i$ по переменной $x$ ) условием порядка $i$ (соответственно, условием порядка $i$ по переменной $x$ ). (При этом значению самого многочлена соответствует $i=0$ ). Линейно упорядочим точки произвольным образом, и частные 
производные так, чтобы не убывал порядок. Используя эти упорядочения, линейно упорядочим все условия по точкам, а при равных точках по частным производным. Будем перебирать условия в соответствии с этим упорядочением и строить искомьй многочлен по шагам. На $i$-м шаге добавляем к ранее построенному многочлену $q_{i}$ такой многочлен $p_{i}$, у которого значения производных во всех ранее рассмотренных условиях равны нулю, а значение производной в $i$-ом условии таково, чтобы это условие выполнялось для $q_{i}+p_{i}$.

Пусть $i$-е условие соответствует точке $a=\left\langle a_{1}, \ldots, a_{k}\right\rangle$, а уже рассмотренные условия - точкам $b_{1}, \ldots, b_{m}$ и, возможно, $a$. Многочлен $p_{i}$ - это некоторое число $c$, умноженное на произведение степеней двучленов вида $x-t$, где $x$ пробегает все переменные, a $t$ для каждой переменной $x_{j}$ пробегает попарно различные значения координаты $x_{j}$, встретившиеся среди координат точек $b_{1}, \ldots, b_{m}, a$. Для $j$-ой координаты точки $b_{n}$, не равной $a_{j}$, берем степень соответствуюшего множителя больше порядка всех условий - это обеспечивает равенство нулю производных $p_{i}$ в условиях, соответствующих этим точкам. Для точки $a$ степень множителя $x_{j}-a_{j}$ берем равной порядку $i$-го условия по переменной $x_{j}$. Во-первых, этим обеспечивается для $p_{i}$ равенство нулю в точке $a$ произвольной производной $d$ в ранее рассмотренных условиях, соответствующих точке $a$. Действительно, так как порядок $d$ не больше порядка $i$-го условия, найдется переменная $x_{j}$, имеющая порядок в $d$ строго меньше, чем в $i$-ом условии. Поэтому, в любом произведении двучленов, естественным образом входящем в качестве слагаемого в $d\left(p_{i}\right)$, степень двучлена $x_{j}-a_{j}$ ненулевая.

Во-вторьх, частная производная $p_{i}$ в $i$-ом условии ненулевая. Действительно, в любом ее слагаемом, где хотя бы раз взятие производной по $x_{j}$ применяется не к соответствующему множителю $x_{j}-a_{j}$, этот множитель присутствует в ненулевой степени и обнуляет это слагаемое. В том же единственном слагаемом, где взятие производных всегда применялось к “своим" множителям, остались лишш множители типа $x_{j}-t$, где $t \neq a_{j}$. Следовательно, рассматриваемая частная производная не равна нулю в точке $a$, и за счет выбора числа $c$ ей можно придать любое требуемое значение. Утверждение доказано.

Автор выражает глубокую признательность Ан. А. Мучнику, беседы с которым способствовали существенному улучшению текста, а также Н. К. Верещагину за ценные замечания.

\section{СПИСОК ЦИТИРОВАННОЙ ЛИТЕРАТУРЫ}

[1] Бахвалов Н. С., ЖКидков Н. П., Кобельков Г. М. Численные методы. М.: Лаборатория базовых знаний, 2001.

[2] Кокс Д., Литтл Дж., О'Ши Д. Идеалы, многообразия и алгоритмы. Введение в вычислительные аспекты алгебраической геометрии и коммутативной алгебры. М.: Мир, 2000.

[3] Верещагин Н. К., Шень А. Х. Языки и исчисления. М.: МЦНМО, 2000.

[4] Шафаревич И. Р. Основы алгебраической геометрии. Т. 1. М.: Наука, 1998.

Институт проблем передачи информации РАН

Поступило

E-mail : gorbunov@iitp.ru

08.11.2001

Исправленный вариант 10.02 .2003 